\title{
Applying Machine Learning Techniques in Older People Activity Recognition using Wearable and Mobile Devices
}

\author{
Flavio Vinicius Vieira Santana, Bruno Henrique Rasteiro, Larissa Cardoso Zimmermann, Luciana \\ De Nardin, Maria da Graça Campos Pimentel \\ Universidade de São Paulo, São Carlos-SP, Brasil \\ flavio.santana@usp.br,bruno.rasteiro@usp.br,larissa.zimmermann@usp.br,lnardin@usp.br,mgp@icmc.usp.br
}

\begin{abstract}
We investigate the potential of the combined use of smartwatch accelerometer data and smartphone apps for online older adultsactivity recognition. We selected machine learning algorithms which resulted in a posteriori recognition accuracy of $98.92 \%$. Our smartphone app, with the selected machine learning algorithms, carried out online recognition from data captured on the smartwatch. These results allow us, as future work, assess the accuracy of online recognition when the system is used by older adults.

Keywords: HAR, smartphone, smartwatch, ML, older people.
\end{abstract}

\section{INTRODUÇÃO}

Reconhecer atividades humanas (HAR: Human Activity Recognition) e identificar (quebra de) padrões comportamentais de indivíduos é um campo de pesquisa promissor e desafiador, com aplicações em segurança, saúde, entretenimento, entre outras [8]. Apesar do crescimento da população idosa em todo o mundo, poucas pesquisas em HAR empregam datasets compostos por atividades realizadas por idosos [3,9] - o que é problemático dado que diferenças significativas foram identificadas, inclusive, entre grupos de idosos [6]

Alguns pesquisadores investigam HAR a partir de dados de sensores de smartphones [6, 7]. Entretanto smartwatches, dotados de acelerômetro, giroscópio, microfone e sensor de batimentos cardíacos, permitem coleta informações de forma contínua e não intrusiva $[2,4]$. Tendo como público-alvo adultos com idade a partir de 60 anos, o objetivo deste trabalho é investigar o potencial do uso combinado do acelerômetro de smartwatches com uma smartphone app para o reconhecimento de um conjunto de atividades humanas (andar, correr, subir e descer escada, sentar e levantar) enquanto a atividade é realizada (online activity recognition).

Utilizando o dataset EARDB [12], com dados capturados de 32 idosos enquanto realizam as atividades selecionadas, implementamos algoritmos de aprendizado de máquina que obtiveram acurácia de reconhecimento das atividades de $98.92 \%$ em processamento $a$ posteriori. Como requisito para realizar o reconhecimento online com usuários idosos, investigamos a possibilidade do reconhecimento ser feito online por uma smartphone app que processa dados capturados do acelerômetro de um smartwatch. Implementamos uma Android app que, utilizando os algoritmos que apresentaram melhor resultado no reconhecimento a posteriori, recebe os dados de um smartwatch e realiza com sucesso o reconhecimento online no

In: XVI Workshop de Trabalhos de Iniciação Científica (WTIC 2019), Rio de Janeiro, Brasil. Anais Estendidos do Simpósio Brasileiro de Sistemas Multimídia e Web (WebMedia). Porto Alegre: Sociedade Brasileira de Computação, 2019.

ISSN 2596-1683

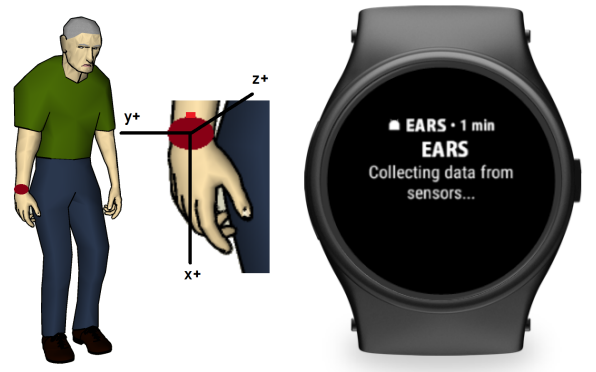

Figura 1: (esq) eixos do acelerômetro; (dir) smartwatch app

smartphone. Em teste realizado com um usuário jovem, a acurácia caiu para $66.9 \%$ - o que era esperado pelo perfil de usuário diferente daquele do EARDB com os quais os algoritmos foram treinados.

Neste texto, a Seção 2 apresenta a EARS Database utilizada, a Seção 3 os trabalhos relacionados, a Seção 4 a metodologia utilizada, a Seção 5 os resultados, e a Seção 6 as considerações finais.

\section{EARDB DATASET}

Para investigar o uso de sensores de smartphones e smartwatches no reconhecimento de atividades de pessoas idosas, Zimmermann [12] desenvolveu um conjunto de aplicativos que permitem a coleta de dados para a criação de datasets associados. Após aprovação do projeto de pesquisa (Plataforma Brasil CAAE: 57875016.3.0000.5390), em colaboração com uma gerontologista foram coletados dados de 15 mulheres e 17 homens com idade entre 60 e 75 anos enquanto realizavam as atividades de andar, correr, subir e descer escada, sentar e levantar - classificadas como atividades simples por Eun et al. [5]. A coleta fez uso de protocolos padronizados na área da saúde (eg Timed Up and Go) enquanto os usuários utilizavam um smartwatch instrumentado para a coleta (Figura 1). A execução de cada atividade foi capturada por 2 minutos; foi utilizado o smartwatch $L G$ Urbane com Android Wear 2.0; foram coletados os dados do acelerômetro de três eixos (Fig 1:esq) com frequência de amostragem de $16 \mathrm{~Hz}$. O correspondente dataset EARDB (Elderly Activity Recognition DataBase) foi utilizado no Elderly Physical Activity Reminder System (EPARS) [13].

\section{TRABALHOS RELACIONADOS}

Capela et al. [1] reportam o estudo de características utilizadas em aplicações HAR baseadas em dispositivos vestíveis e reportadas na literatura. A análise foi realizada de forma a não levar em consideração o classificador utilizado na aplicação. O estudo selecionou 76 características e realizou a análise das mesmas a partir de coleta de dados com um smartphone preso à cintura dos participantes. 
A análise foi feita com três métodos de seleção de características (Relief F, Correlation based Feature Selection e Fast Correlation based Filter), em três populações diferentes e em sete níveis de atividades. No nível de atividade e na população de interesse deste projeto (pessoas acima de 60 anos) foram selecionadas em média 10 características e os recursos do sinal com smartphone nos três grupos de indivíduos (saudáveis, idosos e AVC) foram identificados com sucesso. A aplicação dos três classificadores mostrou ainda que a seleção de um subconjunto de características produziu resultados com precisão bastante semelhante, ou um pouco melhor, do que quando foi utilizado o conjunto completo de características, evidenciando que, o conjunto de características utilizado é independente do classificador. No contexto do trabalho aqui reportado, a principal diferença é com relação a forma com que os dados foram coletados: Capela et al. [1] utilizam smartphone preso à cintura enquanto utilizamos um smartwatch no pulso do participante.

Zhang and Sawchuk [11] selecionaram características de dois tipos: 87 características estatísticas e 23 características físicas. Participaram da coleta de dados 6 indivíduos de diferentes gêneros, idade, altura e peso. O dispositivo utilizado - NotionMode, foi fixado na parte frontal do quadril dos indíviduos. A coleta dos dados foi feita a $100 \mathrm{~Hz}$ utilizando uma janela de tempo de 2 segundos com $50 \%$ de sobreposição. A seleção das características foi feita através de 3 métodos: Relief F, SFC (Wrapper Method based on Single Feature Classification) e SFS (Wrapper Method based on Sequential Forward Selection). Utilizando apenas 5 características estatísticas selecionadas pelo SFS, o classificador SVM com kernel linear apresentou um erro de $12 \%$, considerado um bom resultado. Em relação ao nosso trabalho, Zhang and Sawchuk [11] se diferencia pelo tipo de aparelho, posicionamento do corpo, características dos participantes, frequência de captura e janela de tempo.

Yang et al. [10] reportam estudo específico para avaliação de risco de queda que comparou (a) informações capturadas por smartbands utilizadas durante 7 dias por indivíduos moradores de um residencial para idosos, e (b) dados de uma avaliação padrão utilizada no residencial (RAI-HC: Resident Assessment Instrument-Home Care). Os dados dos dois instrumentos foram analisados a posteriori utilizando os algoritmos logistic regression, decision tree e random forest. Os autores concluíram que os dados do RAI-HC tiveram performance melhor que os dados da smartband, e que o melhor resultado é obtido com a combinação dos dois. Diferentemente do nosso estudo, os autores avaliaram explicitamente o risco de queda, utilizaram o histórico dos pacientes e analisaram dados coletados em ambiente real. Os dados coletados utilizam atividades reconhecidas como repouso e walking, jogging/running a valores do batimento cardíaco obtido durante essas atividades.

\section{CARACTERÍSTICAS: GERAÇÃO E SELEÇÃO}

\subsection{Geração das características}

Esta seção detalha o processo de geração das características e as análises feitas sobre os conjuntos de características obtidos. As características consistem de medidas estatísticas como média e desvio padrão que são utilizadas como entrada para treinar o classificador

Todas as características foram geradas a partir de quatro dados obtidos pelos sensores: aceleração no eixo $X(A x)$, aceleração no eixo $\mathrm{Y}(\mathrm{Ay})$, aceleração no eixo Z (Az) e a aceleração linear (Acc). Tendo
Tabela 1: Características Estatísticas

\begin{tabular}{l|l}
\hline \hline Nome & Descrição \\
\hline \hline mean_Ax, mean_Ay, mean_Az & $\begin{array}{l}\text { Média do sinal nos 3 eixos de aceleraçao } \\
\text { Desvio padrão do sinal nos 3 eixos de } \\
\text { std_Ax, std_Ay, std_Az }\end{array}$ \\
aceleraçao \\
rms_Ax,rms_Ay,rms_Az & $\begin{array}{l}\text { RMaiz média quadrática) do sinal } \\
\text { nos 3 eixos de aceleraçao }\end{array}$ \\
acc & Média da aceleração linear \\
diff & Diferença da aceleração (Ay - Az - Ax) \\
\hline \hline
\end{tabular}

em vista a frequência de $16 \mathrm{~Hz}$ de amostragem do sensor utilizado na coleta dos dados, foi possível então definir uma janela de tempo para sumarizar as instâncias e assim obter as características. Neste trabalho foi utilizado uma janela de 2 segundos o que significa que, a cada 32 instâncias de dados coletados foi calculada e definida uma instância com $\mathrm{N}$ características. No caso da características média (mean), por exemplo, ela consiste no valor médio das 32 instâncias coletadas no intervalo de $16 \mathrm{~Hz}$ para cada um dos eixos.

O processo de geração das características é feito para cada indivíduo e cada atividade, dessa forma se garante que instâncias de indivíduos ou classes distintas não se misturem. A última etapa do processo é a aleatorização (embaralhamento) dos dados obtidos com o objetivo de evitar um possível viés nos dados. As características estatísticas geradas estão especificadas na Tabela 1.

\subsection{Seleção das características}

Considerando as 11 características descritas na Tabela 1, foi realizada uma análise com o objetivo de reduzir a dimensionalidade. Para isso, foram testadas todas as combinações possíveis de características com a condição de que as 3 características de média ficassem sempre juntas, bem como desvio padrão e RMS. Dessa forma, obteve-se os 31 subconjuntos de características identificados na Tabela 2.

A acurácia foi a métrica de avaliação do desempenho para cada subconjunto em um sistema de avaliação cruzada com 10 folds. Os classificadores escolhidos foram Árvore de Decisão e KNN. Uma vez que o algoritmo KNN necessita da definição do parâmetro livre $\mathrm{K}$, que representa o número de vizinhos mais próximos a serem considerados no processo de classificação, uma análise foi realizada onde para cada subconjunto definido: o algoritmo foi executado com o valor de $\mathrm{K}$ variando de 1 a 10 , e o que obteve melhor acurácia foi definido como K padrão. Os resultados são reportados a seguir.

\section{RESULTADOS}

\section{1 Árvore de decisão}

Os resultados obtidos utilizando o algoritmo de Árvore de Decisão podem ser observados na Figura 2, que apresenta no eixo x cada um dos subconjuntos de características identificados na Tabela 2. Cada experimento foi repetido 10 vezes utilizando validação cruzada: os traços verticais no topo das barras indicam o desvio padrão. Observa-se que os diferentes subconjuntos tiveram pouca influência no desempenho do classificador. A melhor acurácia obtida foi de 98.33, utilizando o subconjunto \#20 (características mean_Ax, mean_Ay, mean_Az, std_Ax, std_Ay e std_Az). 


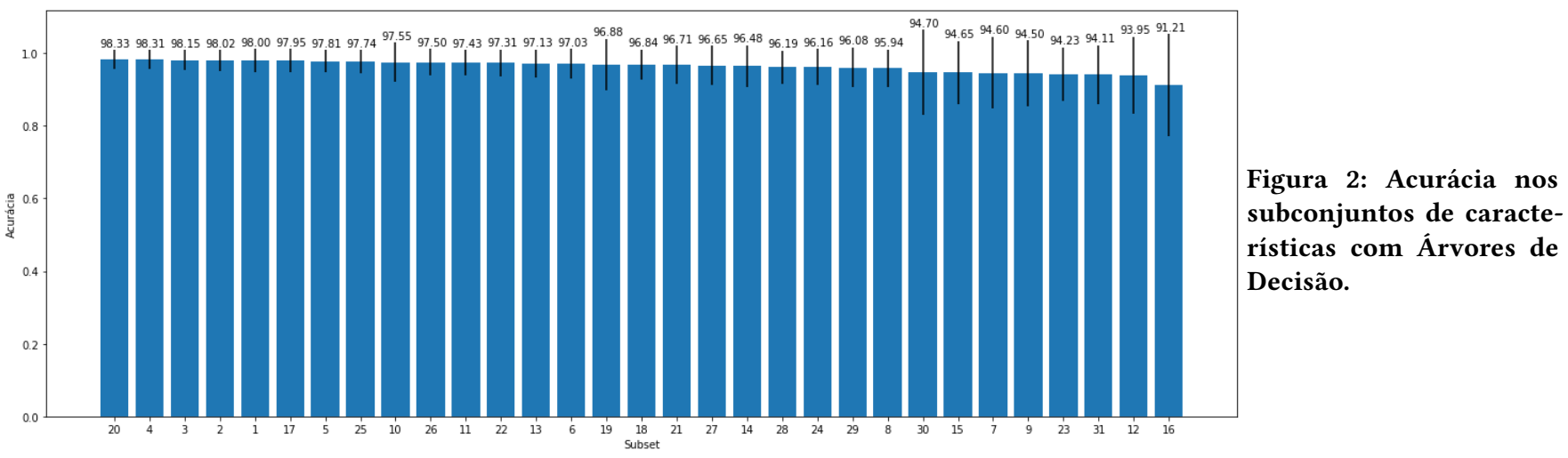

Tabela 2: Subconjuntos (SC) de características estatísticas

\begin{tabular}{|c|c|}
\hline SC & características \\
\hline 1 & $\begin{array}{l}\text { diff, mean_Ax, mean_Ay, mean_Az, std_Ax, std_Ay, std_Az, } \\
\text { rms_Ax, rms_Ay,rms_Az, acc }\end{array}$ \\
\hline 2 & $\begin{array}{l}\text { diff, mean_Ax, mean_Ay, mean_Az, std_Ax, std_Ay, std_Az, } \\
\text { rms_Ax, rms_Ay,rms_Az }\end{array}$ \\
\hline 3 & $\begin{array}{l}\text { diff, mean_Ax, mean_Ay, mean_Az, std_Ax, std_Ay, std_Az, } \\
\text { acc }\end{array}$ \\
\hline 4 & diff, mean_Ax, mean_Ay, mean_Az, std_Ax, std_Ay, std_Az \\
\hline 5 & $\begin{array}{l}\text { diff, mean_Ax, mean_Ay, mean_Az, rms_Ax, rms_Ay, rms_Az, } \\
\text { acc }\end{array}$ \\
\hline 6 & diff, mean_Ax, mean_Ay, mean_Az, rms_Ax, rms_Ay, rmsAz \\
\hline 7 & diff, mean_Ax, mean_Ay, mean_Az, acc \\
\hline 8 & diff, mean_Ax, mean_Ay, mean_Az \\
\hline 9 & diff, std_Ax, std_Ay, std_Az,rms_Ax, rms_Ay, rms_Az, acc \\
\hline 10 & diff, std_Ax, std_Ay, std_Az, rms_Ax, rms_Ay, rms_Az \\
\hline 11 & diff, std_Ax, std_Ay, std_Az, acc \\
\hline 12 & diff, std_Ax, std_Ay, std_Az \\
\hline 13 & diff, rms_Ax, rms_Ay, rms_Az, acc \\
\hline 14 & diff, rms_Ax, rms_Ay, rms_Az \\
\hline 15 & diff, acc \\
\hline 16 & diff \\
\hline 17 & $\begin{array}{l}\text { mean_Ax, mean_Ay, mean_Az, std_Ax, std_Ay, std_Az, } \\
\text { rms_Ax, rms_Ay, rms_Az, acc }\end{array}$ \\
\hline 18 & $\begin{array}{l}\text { mean_Ax, mean_Ay, mean_Az, std_Ax, std_Ay, std_Az, } \\
\text { rms_Ax, rms_Ay,rms_Az }\end{array}$ \\
\hline 19 & mean_Ax, mean_Ay, mean_Az, std_Ax, std_Ay, std_Az, acc \\
\hline 20 & mean_Ax, mean_Ay, mean_Az, std_Ax, std_Ay, std_Az \\
\hline 21 & mean_Ax, mean_Ay, mean_Az, rms_Ax, rms_Ay, rms_Az, acc \\
\hline 22 & mean_Ax, mean_Ay, mean_Az, rms_Ax, rms_Ay, rms_Az \\
\hline 23 & mean_Ax, mean_Ay, mean_Az, acc \\
\hline 24 & mean_Ax, mean_Ay, mean_Az \\
\hline 25 & std_Ax, std_Ay, std_Az, rms_Ax, rms_Ay, rms_Az, acc \\
\hline 26 & std_Ax, std_Ay, std_Az, rms_Ax, rms_Ay, rms_Az \\
\hline 27 & std_Ax, std_Ay, std_Az, acc \\
\hline 28 & std_Ax, std_Ay, std_Az \\
\hline 29 & rms_Ax, rms_Ay, rms_Az, acc \\
\hline 30 & rms_Ax, rms_Ay, rms_Az \\
\hline 31 & acc \\
\hline
\end{tabular}

\section{$5.2 \mathrm{KNN}$}

O algoritmo 1NN obteve maior acurácia para todos os subconjuntos de características (Figura 3). A Figura 4 mostra a acurácia para todos os subconjuntos: a melhor acurácia (98.92) foi do subconjunto \#4 (diff, mean_Ax, mean_Ay, mean_Az, std_Ax, std_Ay, std_Az).
Tabela 3: Acurácia online dos classificadores (usuário jovem)

\begin{tabular}{|l|c|c|c|}
\hline \hline \multirow{2}{*}{ Atividade } & \multicolumn{3}{|c|}{ Acurácia } \\
\cline { 2 - 4 } & 1NN (\%) & 3NN (\%) & 5NN (\%) \\
\hline \hline Sentar & 100.0 & 100.0 & 100.0 \\
\hline Ficar em pé & 96.7 & 96.7 & 100.0 \\
\hline Andar & 70.0 & 66.7 & 40.0 \\
\hline Descer escada & 11.1 & 40.7 & 46.9 \\
\hline Subir escada & 20.5 & 30.3 & 14.3 \\
\hline \hline Média & 59.7 & 66.9 & 60.2 \\
\hline \hline
\end{tabular}

\subsection{Online activity recognition}

Para medir a acurácia do classificador no reconhecimento online de atividades, foi desenvolvida uma extensão (Figura 5) para um aplicativo de coleta de dados de Zimmermann [12]. Considerando os resultados reportados na seção anterior, na extensão foi implementado o algoritmo KNN com as características diff, mean_Ax, mean_Ay, mean_Az, std_Ax, std_Ay e std_Az (subconjunto \#4) para classificar as atividades de sentar, ficar em pé, andar, descer escada e subir escada. Foi utilizado um smartphone Motorola Moto G6 Play.

Um usuário (um dos autores, aluno de graduação de 22 anos) utilizou um smartwatch LG Urbane com Android Wear 2.0 para realizar cada uma das atividades por 1 minuto. Como a janela de tempo é de 2 segundos, tivemos 30 classificações feitas por atividade e o desempenho obtido está expresso na Tabela 3 para $n=1,3$ e 5 .

A Tabela 3 indica que os três classificadores tiveram acurácia alta nas atividades de sentar e ficar em pé enquanto que o $3 \mathrm{NN}$ teve o melhor desempenho médio. As atividades de descer e subir escadas tiveram acurácia baixa: o classificador recorrentemente reconheceu essas atividades como "andar". Essa confusão pode estar associada à combinação do posicionamento do smartwatch, no pulso, com o movimento dos braços (up and down) durante essas atividades.

Comparados à acurácia reportada na seção anterior, a média de acerto caiu de $98.92 \%$ para $66.9 \%$. Uma das razões para essa variação é que o teste foi realizado com um usuário de perfil diferente (jovem universitário) dos que compõe a EARDB (32 idosos de 60 a 75 anos). Assim, não é possível interpretar esse resultado como um indicativo da precisão do classificador. Por outro lado, os resultados indicam ser possível realizar no smartphone o reconhecimento online das atividades a partir dos dados coletados no smartwatch. 


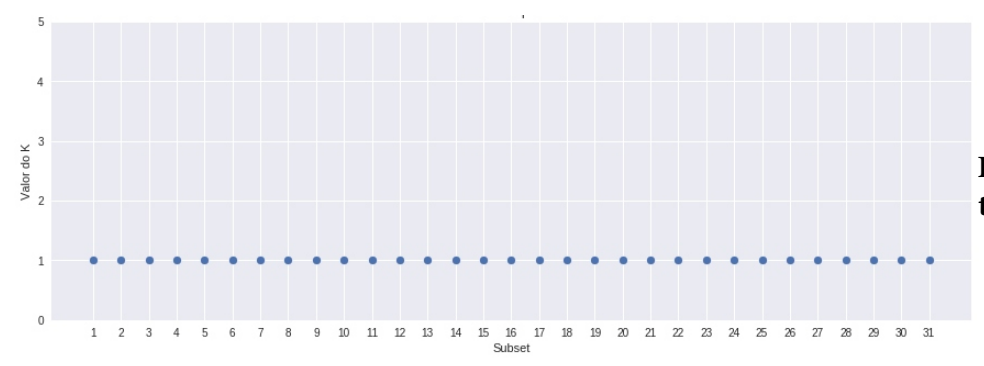

Figura 3: Melhor valor de K para subconjuntos de características.
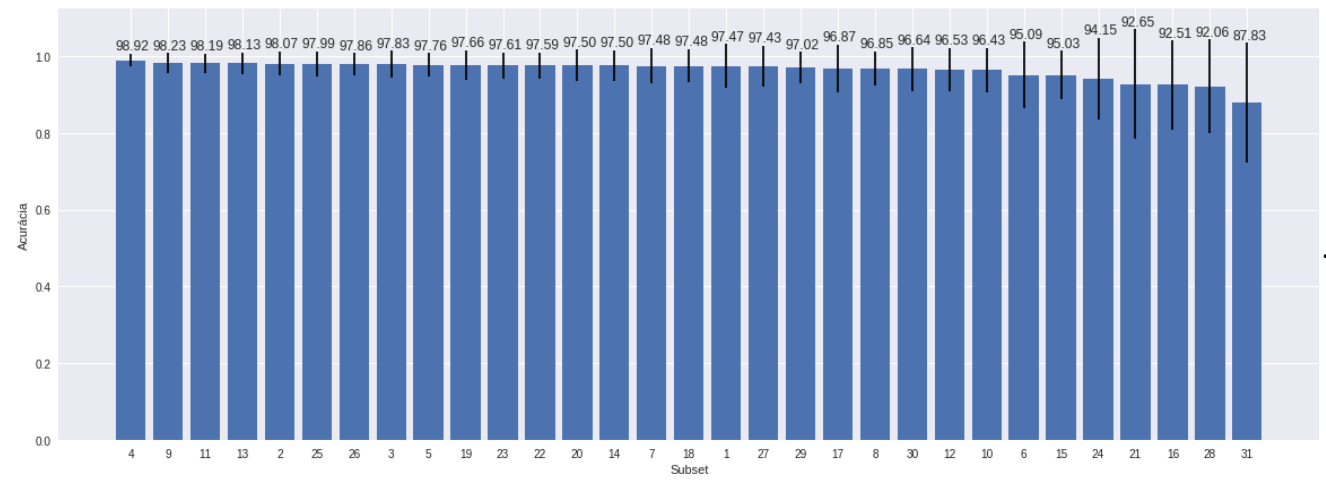

Figura 4: Acurácia nos subconjuntos de características com KNN.

ActivityRecognition

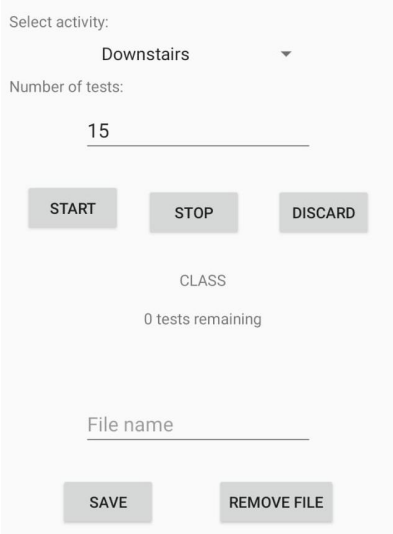

Figura 5: Tela do protótipo para testes dos classificadores

\section{CONSIDERAÇÕES FINAIS}

O trabalho investigou o potencial do uso de dados capturados do acelerômetro de um smartwatch e processados em um smartphone para o reconhecimento online de atividades realizadas por idosos. Algoritmos de machine learning executados a posteriori resultaram em acurácia de reconhecimento de $98.92 \%$. Os algoritmos foram implementados em uma smartphone app e um estudo permitiu realizar, no smartphone, o reconhecimento online das atividades a partir dos dados capturados no smartwatch. Esses resultados abrem portas para trabalhos futuros: o próximo passo é avaliar a acurácia do reconhecimento online com usuários idosos.
Agradecimentos: Agradecemos pelo financiamento: Santana (CNPqPIBIC); Rasteiro (PUB-USP); Zimmermann (MSc CAPES); De Nardin (Programa Permanente de Capacitação Docente PUC Minas); Pimentel (CNPq-PQ; FAPESP 16/00351-6 e 16/50489-4).

\section{REFERÊNCIAS}

[1] Capela, N. A., Lemaire, E. D., and Baddour, N. (2015). Feature selection for wearable smartphone-based human activity recognition with able bodied, elderly, and stroke patients. PloS one, 10(4):e0124414.

[2] Davoudi, A. et al. (2019). Accuracy of Samsung Gear S Smartwatch for Activity Recognition: Validation Study. 7MIR Mhealth Uhealth, 7(2):e11270.

[3] De-La-Hoz-Franco, E. et al. (2018). Sensor-based datasets for human activity recognition: A systematic review of literature. IEEE Access, 6:59192-59210.

[4] Dorn, D., Gorzelitz, J., Gangnon, R., Bell, D., Koltyn, K., and Cadmus-Bertram, L. (2019). Automatic identification of physical activity type and duration by wearable activity trackers: A validation study. FMIR Mhealth Uhealth, 7(5):e13547.

[5] Eun, S.-J., Whangbo, T.-K., Park, D. K., and Kim, K.-H. (2017). Development of personalized urination recognition technology using smart bands. International neurourology journal, 21(Suppl 1):S76.

[6] Galán-Mercant, A. and Cuesta-Vargas, A. I. (2013). Differences in trunk accelerometry between frail and nonfrail elderly persons in sit-to-stand and stand-to-sit transitions based on a mobile inertial sensor. FMIR Mhealth Uhealth, 1(2):e21.

[7] Jansi, R. and Amutha, R. (2019). Sparse representation based classification scheme for human activity recognition using smartphones. Multimedia Tools Appl., 78(8):11027-11045.

[8] Lara, O. D. and Labrador, M. A. (2012). A survey on human activity recognition using wearable sensors. IEEE communications surveys \& tutorials, 15(3):1192-1209. [9] Lentzas, A. and Vrakas, D. (2019). Non-intrusive human activity recognition and abnormal behavior detection on elderly people: a review. Artificial Intelligence Review. [10] Yang, Y., Hirdes, J., et al. (2019). Fall risk classification in community-dwelling older adults using a smart wrist-worn device and the resident assessment instrumenthome care: Prospective observational study. FMIR Aging, 2(1):e12153.

[11] Zhang, M. and Sawchuk, A. A. (2011). A feature selection-based framework for human activity recognition using wearable multimodal sensors. In Proceedings of the 6th International Conference on Body Area Networks, pages 92-98. ICST.

[12] Zimmermann, L. C. (2019). Reconhecimento de atividades de pessoas idosas com integração de sensores de smartphones e dispositivos vestiveis. Master's thesis, PPg-CCMC, USP/São Carlos, Brasil https://doi.org/10.11606/D.55.2019.tde-10062019-080049. [13] Zimmermann, L. C., Rodrigues, K. R., and Pimentel, M. G. C. (2019). EPARS: Elderly Physical Activity Reminder System Using Smartphone and Wearable Sensors. http://doi.acm.org/10.1145/3341162.3350845. In Adjunct Proceedings UbiComp/ISWC'19, pages 1139-1145. 